\title{
Gingivoplastía con láser de diodo de alta intensidad por agrandamiento gingival
}

\author{
Gingivoplasty with high-intensity diode laser for gingival enlargement
}

Antonio Isaac Muñoz Lara ${ }^{1 a}$, María José Jarrín Peñafiel ${ }^{1 a}$, Juan Pablo Jaramillo Burneo ${ }^{2 b}$

\section{RESUMEN}

El articulo reporta el caso de un paciente masculino de 53 años de edad, quien acudió a la Clínica de Servicios Odontológicos Universitarios (SERODU), en Ecuador, por presentar sonrisa gingival. No presentaba antecedentes médicos relevantes. Se le diagnosticó agrandamiento gingival, de las piezas dentarias 1.1,1.2 y 2.1 y fue tratado con gingivoplastía con láser de alta intensidad de diodo. A los 15 días de control se observó una buena cicatrización y el paciente manifestó no haber tenido dolor posoperatorio.

El objetivo de este caso clínico, es mostrar los beneficios del láser de diodo de alta intensidad, para el tratamiento del agrandamiento gingival y con ello lograr una mejor percepción estética de los dientes antero superiores.

PALABRAS CLAVE: Gingivoplastia; Hiperplasia Gingival; Terapia por láser

\section{ABSTRACT}

The article reports the case of a 53-year-old male patient, who went to the Clinic of University Dental Services (SERODU), in Ecuador, for presenting a gingival smile. He had no relevant medical history. He was diagnosed with a gingival enlargement of teeth 1.1, 1.2 and 2.1 and was treated with high intensity diode laser gingivoplasty. After 15 days of control, good healing was observed and the patient stated that he had no postoperative pain.

The objective of this clinical case is to show the benefits of the high intensity diode laser, for the treatment of gingival enlargement and thereby achieve a better aesthetic perception of the upper anterior teeth.

KEY WORDS: Gingivoplasty; Gingival Hyperplasia; Laser therapy. 


\section{INTRODUCCIÓN}

La odontología evoluciona constantemente ${ }^{(1)}$; sin embargo, el objetivo de rehabilitación del sistema dentofacial de manera funcional se combina con los requerimientos estéticos deseados por el paciente ${ }^{(2)}$, y la localización de los tejidos blandos circundantes ${ }^{(3)}$.

Cuando es necesario acondicionar los tejidos periodontales circundantes por razones estéticas, en donde se aprecian coronas clínicas cortas combinadas con la presencia de línea de sonrisa alta, generando exposición de gran cantidad de encía al hablar o sonreír, es posible recurrir al alargamiento coronario ${ }^{(4)}$, definido como el incremento en la longitud de la corona clínica mediante procedimiento resectivo de encía conocido como gingivoplastía con o sin osteotomía, el cual permite modificar el espacio biológico ${ }^{(5)}$, que es un procedimiento quirúrgico conservador que permite realizar cortes tisulares específicos a nivel de la mucosa para reposicionarla ${ }^{(6)}$.

Este procedimiento quirúrgico convencional con uso de bisturí, pueden generar rechazo por el paciente, debido al tiempo de intervención prologando y el curso del postoperatorio (7); sin embargo, en el campo odontológico actual, con el advenimiento de la terapia láser de alta intensidad y su aplicación en procedimientos quirúrgicos, se logra controlar estos factores con una técnica poco invasiva, menos dolorosa, efectiva, rápida, precisa y segura desde el punto de vista biológico, generando mayor interés y expectativas por parte del paciente ${ }^{(8)}$, pues sin duda puede ser la opción de un tratamiento que genera efectos favorables en menor tiempo y constituye una propuesta tentadora $^{(9)}$.

En el caso reportado, se observó la presencia de un agrandamiento gingival en las piezas dentarias 11,12 y 21 razón por la cual se realizó la gingivoplastía con láser de diodo de alta intensidad.

\section{Reporte del caso}

Paciente de 53 años de edad, de sexo masculino, acude a la clínica de Servicios Odontológicos Universitarios (SERODU), en Quito, Ecuador, manifestando que se sentía disconforme con su sonrisa, percibía sus dientes de menor tamaño y al hablar o sonreír exponía encía. No refiere antecedentes médicos relevantes.

Al examen clínico intraoral se observa un ligero agrandamiento gingival en dientes antero superiores. Se realiza, un periodontograma, sin evidencia de mayores alteraciones periodontales. (Figura 1A). Se llegó al siguiente diagnóstico: Deformidades y condiciones mucogingivales alrededor de los dientes: "agrandamiento gingival"
El plan de tratamiento fue: profilaxis, gingivoplastia con láser de alta intensidad de diodo. Reevaluación y mantenimiento.

\section{Tratamiento}

Se realizó una profilaxis con ultrasonido al paciente. Para el procedimiento quirúrgico se colocó anestésico tipo lidocaína al $10 \%$ en spray en la mucosa a nivel de encía papilar de los órganos dentarios 1.1, 1.2 y 2.1 , se infiltró con clorhidrato de mepivacaína con adrenalina al $2 \%$, a nivel de fondo de surco para bloquear el nervio alveolar superior anterior, utilizando una cantidad de $0,9 \mathrm{ml}$ del tubo, cantidad que fue considerada suficiente para este procedimiento. (Figura 2A).

Se realizó la medición con una sonda periodontal de las piezas a una distancia adecuada por debajo del margen gingival, que permitiera llegar al límite amelocementario, para determinar a qué nivel se realizaría la incisión de la encía y de esta manera mantener la uniformidad del zenit gingival de las piezas dentales a tratar con respecto las piezas adyacentes. (Figura 2B).

Una vez establecido el sitio de corte se direccionó al haz de luz del láser de diodo de alta intensidad de forma biselada, ligeramente angulada siguiendo el contorno gingival desde la línea media hacia la hemiarcada derecha, y posteriormente hacia la hemiarcada izquierda. (Figura 2C, 2D).

Luego de hacer la incisión con el láser de diodo, se verificó si se realizó a la distancia establecida en un principio. (Figura 2E, 2G)

Se entregó por escrito la receta médica e indicaciones postoperatorias. Se tomó una fotografía postoperatoria (3B).

\section{Reevaluación y mantenimiento}

A los 15 días de control, al examen clínico se observó tejido de cicatrización (Figura 3C). El paciente no refirió percepción dolorosa durante el curso del postoperatorio; además refirió comodidad durante la función y conformidad con los resultados estéticos obtenidos con el procedimiento quirúrgico.

Al evaluar el borde libre de encía marginal se apreció reposición apical a una distancia de aproximadamente 0,5 a $1 \mathrm{~mm}$ de la línea amelocementaria en los órganos dentarios 1.1, 1.2 y 2.1. Esta es otra de las ventajas, ya que durante el procedimiento quirúrgico se logra precisar hasta dónde se desea retirar el tejido marginal, respetando desde luego el espacio biológico. 

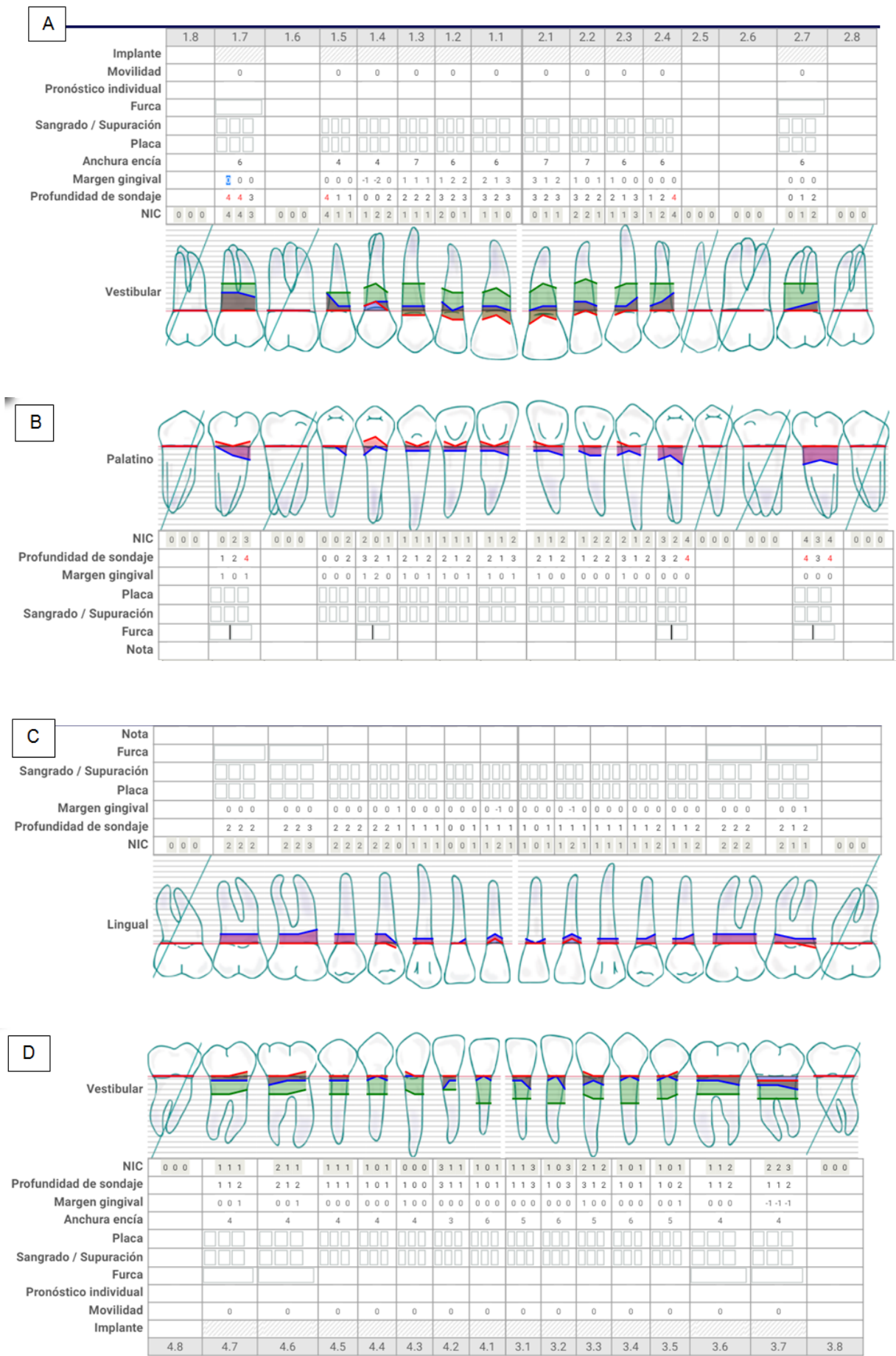

Figura 1. A. Periodontograma de arcada superior por vestibular. B. Periodontograma de arcada superior por palatino. C. Periodontograma de arcada inferior por vestibular. D Periodontograma de arcada inferior por lingual postoperatoria después de 15 días 

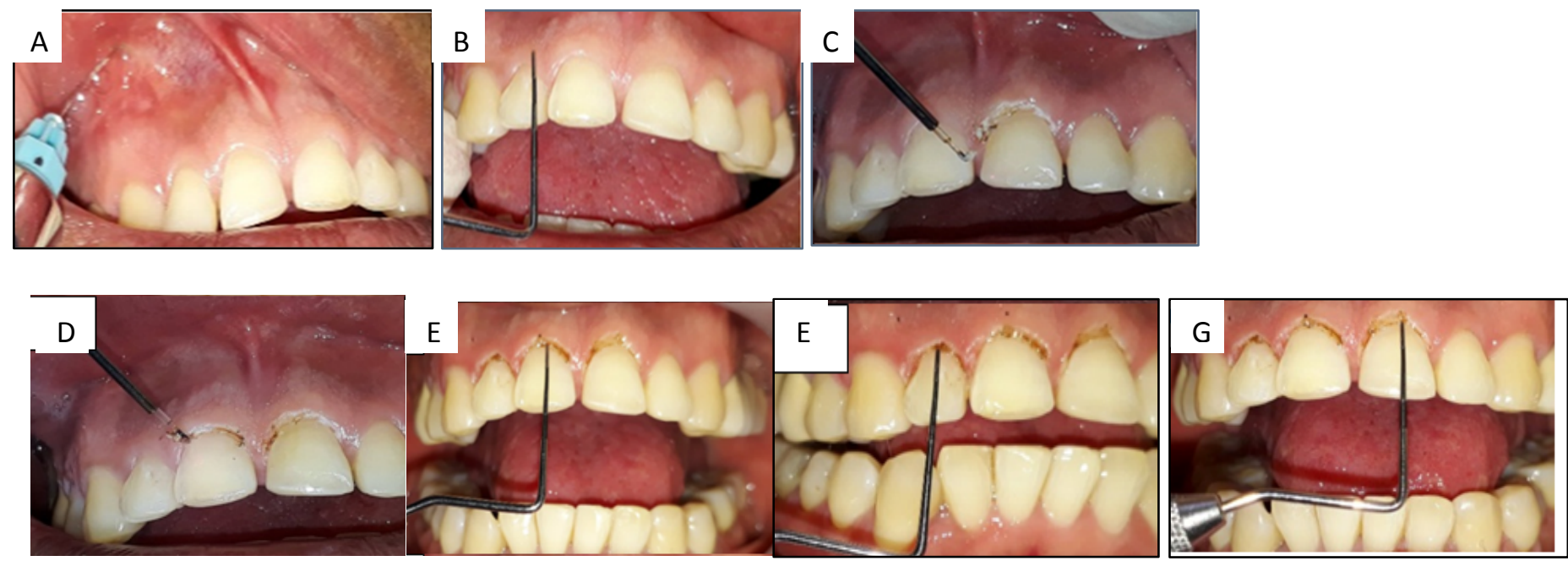

Figura 2.A. Bloqueo anestésico del nervio alveolar anterior. B. Medición con una sonda periodontal de la pieza número 21 a qué nivel se realizará el corte de la encía. C. Gingivoplastia de la pieza número 21.D Gingivoplastia de la pieza número 12. E,F,G Se verifica si la incisión se realizó a la distancia establecida

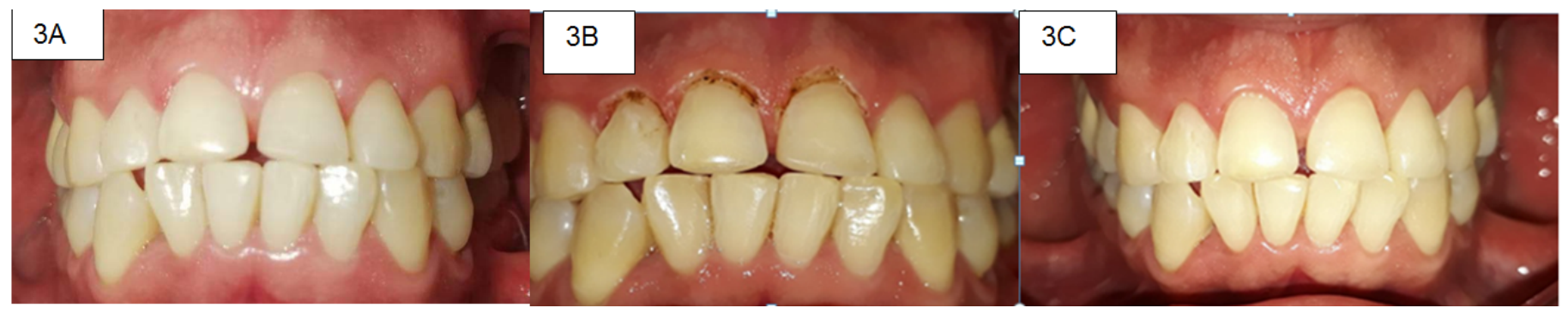

Figura 3.A.Foto Inicial 3B. Al finalizar la gingivoplastia. 3C. Fotografía postoperatoria después de 15 días

\section{DISCUSIÓN}

Al utilizar el láser de alta intensidad en procedimientos quirúrgicos, el paciente experimenta menos dolor postoperatorio ${ }^{(11)}$; además la dosis de analgésicos que se prescribe para el control del dolor postoperatorio suele ser menor en comparación con las dosis utilizadas con otras técnicas como la convencional con bisturí ${ }^{(12)}$.

Durante la consulta odontológica la colocación de la anestesia es uno de los procedimientos que puede convertirse en un factor estresante, por lo que se hace necesario considerar la necesidad y cantidad de anestésico en el momento de intervenir quirúrgicamente con láser o con bisturí en tejidos periodontales ${ }^{(13)}$.

En la literatura se reporta que la necesidad de anestesia infiltrativa en cirugía con láser de alta intensidad es poca o nula ${ }^{(14)}$, ya que solo con anestésico tópico se puede realizar el procedimiento, lo

cual es importante para minimizar la experiencia traumática, especialmente en pacientes pediátricos ${ }^{(15)}$.

Otra de las ventajas de utilizar láser de alta intensidad es la reducción del sangrado y el tiempo de cicatrización ${ }^{(15)}$, así como la posibilidad de realizar el procedimiento en un campo operatorio con cantidad disminuida de microorganismos debido a la actividad antimicrobiana del láser ${ }^{(16)}$.

También se refiere que al realizar alargamiento coronal con láser de diodo, la recuperación es en menor tiempo, lo que puede favorecer los procedimientos restaurativos y protésicos ${ }^{(17)}$.

La recurrencia del tejido después de la gingivoplastía es frecuente ${ }^{(18)}$; sin embargo, al comparar la técnica láser con la convencional la literatura refiere que después de un seguimiento de 6 meses existe menor recurrencia en la gingivoplastía realizada con láser de alta intensidad 20$)$ 
La recurrencia del tejido después de la gingivoplastía es frecuente ${ }^{(18)}$; sin embargo, al comparar la técnica láser con la convencional la literatura refiere que después de un seguimiento de 6 meses existe menor recurrencia en la gingivoplastía realizada con láser de alta intensidad $^{(19,20)}$.

\section{CONCLUSIÓN}

En el manejo del caso clínico actual se puede concluir que la terapia con láser de alta intensidad, constituye una buena alternativa a la técnica convencional, ya que el láser de alta intensidad proporciona una incisión limpia, reduce la hemorragia por medio de un sellado de pequeños vasos sanguíneos y linfáticos que dan como resultado una hemostasia, incluso el dolor postoperatorio utilizando esta técnica es reducido o casi nulo ya que sella los haces nerviosos durante el corte.

Contribuciones de autoría: AIML, MJJP, JPJB: Participaron en el diagnostico, tratamiento, revisión de la información, redacción y aprobación del artículo.

Fuente de financiamiento: Autofinanciado.

Conflictos de interés: No declararon conflictos de interés.

\section{REFERENCIAS BIBLIOGRÁFICAS}

1. Rosa M, Olimpo A, Fastuca R, Caprioglio A. Perceptions of dental professionals and laypeople to altered dental esthetics in cases with congenitally missing maxillary lateral incisors. Prog Orthod. 2013; 14:34

2. Sobouti F, Rakhshan V, Chiniforush N, Khatami M. Effects of laser-assisted cosmetic smile lift gingivectomy on postoperative bleeding and pain in fixed orthodontic patients: a controlled clinical trial. Prog Orthod. 2014; 15:66.

3. Wilckens $M$, Beltrán V, Leiva $C$, Donaire F. Manejo quirúrgico periodontal de la erupción pasiva alterada: reporte de casos. Rev. Clin. Periodoncia Implantol. Rehabil. Oral [Internet]. $2015 \quad$ Ago [citado 2019 Oct 26]; 8(2): 167-172. Disponible en: https://scielo.conicyt.cl/scielo.php?script=sci_arttext\&pid= S071901072015000200012\&lng=es. http:///dx.doi.org/10. 1016/j.piro.2015.03.003

4. Manzur-Villalobos I, Díaz-Rengifo IA, Manzur-Villalobos D, Díaz-Caballero AJ. Agrandamiento gingival farmacoinducido. Univ. Salud. 2018; 20(1):89-96.
5. Noviello A Bisturí contra gingivectomía con láser en pacientes ortodónticos en el tratamiento de la salud periodontal. Roma Tor Vergata. 2018;(5):16.

6. Soriano $R$, Cáceres $A$. Resolución del agrandamiento gingival mediante terapia periodontal no quirúrgica: reporte de caso. Rev. Odont. Mex. 2016; 20 (4): 253-258

7. Dorina L. Lucchese A. Di Stasio D. Molecular Aspects of Drug-Induced Gingival Overgrowth: An In Vitro Study on Amlodipine and Gingival Fibroblast.Int J Mol Sci. 2019; 20(8).

8. Ceccarelli JF, Ricaldi J, Berastain JF. Fibromatosis gingival. Diagnóstico y tratamiento: Reporte de un caso. Rev Estomatol Herediana.2010; 20(3):161-165.

9. Malek R, El Houari B, Kissa J. Manejo periodontal del sobrecrecimiento gingival inducido por ciclosporina $A$ : un enfoque no quirúrgico. Caso Rep Dent.2019;(10):55.

10. Heitz LJ, Lang NP. Surgical and nonsurgical periodontal therapy. Learned and unlearned concepts. Periodontol. 2013; 62(1):218-231.

11. Shukla $P$, Dahiya V, Kataria $P$, Sabharwal $S$. Inflammatory hyperplasia: From diagnosis to treatment. J Indian Soc Periodontol. 2014; 18; 92-4.

12. Alvarado A, Ramírez S, Nieto A, García R. Alargamiento de corona estético previo a rehabilitación protésica. Informe de caso. Rev. Clin. Periodoncia Implantol. Rehabil. Oral [Internet]. 2018 Dic [citado 2019 Jul 17]; 11( 3 ):170-172.

13. Al-Mohaya MA, Al-Malik AM. Escisión de granuloma piógeno oral en un paciente diabético con láser de diodo de $940 \mathrm{~nm}$. Arabia Saudita Med .2016; 37(12): 13951400.

14. Azma E, aplicación de láser Safavi N. Diode en cirugía oral de tejidos blandos.J Lasers Med Sci .2013; 4(4): 206-11.

15. Subramani, T, Rathnavelu V, Alitheen NB, Padmanabhan P. Cellular crosstalk mechanism of Tolllike receptors in gingival overgrowth (review). Int $\mathrm{J}$ Mol Med. 2015; 35(5); 1151-8.

16. Convissar, R. Láser en Odontología. Principios y práctica. España: Elsevier;2014.

17. Trullols, C., et. al. Aplicaciones del láser blando en odontología. Anales de Odontoestomatología. 1997;(2):45-51.

18. Oltra, D., et al. Aplicaciones del láser de baja potencia en Odontología. RCOE. 2004; 9(5):517-524

19. Bennani V, Ibrahim $H$. The periodontal restorative interface. Esthetic considerations. Peridontol 2017; (1):74-101.

20. Liu X, Yu J. Zhou J. Digitally guided dual technique for both gingival and bone resection during Crown lengthening surgery. J Prosthet Dent Mar. 2017; (3):345349.

21. Mostafa D. A succeccful management of sever gummy smile using gingivectomy and botulinum toxin injection. Acase report. International Journal of Surgery Case. $2018 ;(42): 169-174$. 